\title{
Impaired vascular reactivity in sepsis - a systematic review with meta-analysis
}

Sigita Kazune ${ }^{1}$, Anda Piebalga ${ }^{2}$, Eva Strike ${ }^{1}$, Indulis Vanags ${ }^{1}$

${ }^{1}$ Department of Anaesthesiology and Intensive Care, Riga Stradins University, Riga, Latvia ${ }^{2}$ Department of Toxicology and Sepsis, Riga East University Hospital, Riga, Latvia

Submitted: 22 April 2019

Accepted: 12 May 2019

Arch Med Sci Atheroscler Dis 2019; 4: e151-e161

DOI: https://doi.org/10.5114/amsad.2019.86754

Copyright (c) 2019 Termedia \& Banach

\author{
Corresponding author: \\ Sigita Kazune \\ Department of \\ Anaesthesiology \\ and Intensive Care \\ Riga Stradins University \\ 13 Pilsonu St \\ LV-1002 Riga, Latvia \\ E-mail: sigita.kazune@rsu.lv
}

\begin{abstract}
Introduction: Vascular dysfunction due to reduced nitric oxide bioavailability plays an important role in the pathogenesis of sepsis. This meta-analysis examines evidence from published literature to evaluate whether in the adult population the presence/severity of sepsis is associated with impaired vasoreactivity.

Material and methods: We performed a search of the Medline, Scopus, and EMBASE databases to identify observational studies using measurement of reactive hyperaemia in adult patients with sepsis. After data extraction using predefined protocol, qualitative synthesis of findings was performed regarding consistency of findings between methods, evidence of association between vascular reactivity and severity of sepsis, multiple organ failure, and death. A meta-analyses of standardised mean differences in vasoreactivity between groups was performed, in which data were available for relevant outcomes.

Results: Eighteen studies using four methods to measure vascular reactivity from a total of 466 were included in the analysis. The pooled standardised mean difference estimate showed that septic patients had less reactive hyperaemia than controls $(-2.59,95 \% \mathrm{Cl}:-3.46$ to $-1.72 ; p<0.00001)$, and peak hyperaemic blood flow was lower in patients with sepsis than in the control group (SMD $=-1.42,95 \% \mathrm{Cl}:-2.14$ to $-0.70 ; p=0.0001)$. The combined SMD between non survivors and survivors was $-0.36(95 \% \mathrm{Cl}:-0.67$ to $-0.06 ; p=0.02)$ for reactive hyperaemia and $-0.70(95 \% \mathrm{Cl}:-1.13$ to -0.27 ; $p=0.001$ ) for peak hyperaemic blood flow.

Conclusions: Septic patients have attenuated vascular reactivity when compared to healthy volunteers. There are insufficient data indicating that these changes can identify patients at risk of worsening organ failure or death.
\end{abstract}

Key words: sepsis, meta-analysis, reactive hyperaemia.

\section{Introduction}

Vascular endothelium is thought to be the key organ involved in the pathogenesis of host response in sepsis [1, 2]. Quantification of endothelial dysfunction may be useful in assessing the potential for development of microvascular perfusion abnormalities and associated organ dysfunction.

One of the main functions of vascular endothelium is control of arterial tone. Activation of endothelium in sepsis is an adaptive response to lipopolysaccharides and cytokines, which leads to altered vasomotor tone and increased blood flow to infected areas $[3,4]$. Activation of vascular 
endothelium may also trigger altered haemodynamic response and microvascular perfusion abnormalities [5].

Several narrative reviews $[1,4,6]$ reported the effects of sepsis on endothelial function, and one systematic review [7] focused on biomarkers of endothelial activation in sepsis. Measurement of reactive hyperaemia to ischaemic or pharmacological stimuli, which serves as a test of endothelial nitric oxide (NO) bioavailability, may be used to quantify endothelial dysfunction [8]. Different stimuli and measurement methodologies have been employed to investigate endothelial function in patients with sepsis with variable results. However, measurement of reactive hyperaemia in sepsis remains a research tool, and association between altered vascular reactivity and severity of sepsis with respect to development of multiple organ failure and mortality is not well established.

The objective of this study was to conduct a systematic review and meta-analysis of the currently published literature to provide a summary of existing research and evaluate whether in the adult population the presence/severity of sepsis is associated with conduit artery and microvascular dysfunction as measured by provocation tests.

\section{Material and methods}

This systematic review follows recommendations of the Preferred Reporting Items for Systematic Reviews and Meta-Analyses (PRISMA) statement [9]. The study was registered at PROSPERO (CRD42018107129). We included English language publications that met the following inclusion criteria: included adult patients (18 years old or over) who were diagnosed with sepsis, severe sepsis, or septic shock, reported measurements of endothelial vascular reactivity by a validated methodology utilising standardised protocols, and incorporated clinical end-points. Primary outcome was changes of endothelial vascular reactivity in relation to the presence or severity of sepsis. Secondary outcomes were development or severity of single or multiple organ failure and intensive care unit, hospital, or 28-day mortality (whichever was available). Studies were excluded if they reported only blood biomarker measurements of endothelial function, included paediatric patients, or were duplicated.

The Medline, Scopus, and EMBASE records from January 1985 to November 2018 were searched to identify relevant studies. A preliminary search using the terms "endothelium" or "endothelial function" in combination with "vascular reactivity", "blood vessel reactivity", "vascular dysfunction", "vascular occlusion test", or "reactive hyperaemia/ hyperaemia" was carried out to identify validated methods for assessment of endothelial function.
Final search terms included "venous occlusion plethysmography", "flow-mediated dilation", "peripheral arterial tonometry", "laser Doppler flowmetry", "pulse wave velocity", and "augmentation index", combined with "sepsis", "septicaemia/ septicemia" or "systemic inflammatory response syndrome". Filters were used to restrict results to human studies and English language publications. References from selected studies were checked to identify any additional articles.

Two researchers independently screened titles and abstracts of all articles retrieved by the search and then examined the full text of the relevant articles for fulfilment of inclusion criteria.

The following data were extracted independently by two researchers: study design, sample size, case mix descriptors (age, comorbidities), sepsis definition used, method, protocol and timing of vasoreactivity evaluation, use of blood-derived markers of endothelial function, group differences in reactive hyperaemia and peak hyperaemic flow related to any clinical outcome (presence or absence of sepsis, single or multiple organ dysfunction, severity of sepsis, mortality), and possible confounders. The definition of sepsis was used as given in the retrieved articles. Risk of random error in vasoreactivity measurement was assessed by patient sample size and reported confidence intervals. To perform risk of bias assessment for this systematic review we adapted the Newcastle-Ottawa scale for case-control studies [10]. This scale evaluates the risk of bias based on group selection, comparability, and assessment of outcome. The risk of bias assessment using the customised scale was done by two researchers independently.

Meta-analyses were performed using Review Manager software (Version 5.3; Cochrane Community). For data reported as median and interquartile range, mean and $95 \%$ confidence interval or, provided for two septic subgroups, mean and standard deviation were estimated using methods described in the Cochrane Handbook for Systematic Reviews [11]. Because vasoreactivity was measured using several different methods, studies were summarised using standardised mean differences (SMD) of the mean of reactive hyperaemia/peak hyperaemic flow between groups for each relevant clinical outcome. Pooled SMD and 95\% confidence interval $(\mathrm{Cl})$ for each measure of vasoreactivity and outcome were obtained using a random effects model for continuous outcomes [12]. Pooled SMD was considered significant if $p<0.05$. Heterogeneity was assessed using Higgins $l^{2}$ test [13]. Significant heterogeneity was assumed if the $R^{2}$ statistic was greater than or equal to $75 \%$.

Planned subgroups to be analysed for heterogeneity included different methods of vasoreactivity assessment and studies using controls at risk 
of endothelial dysfunction. We performed leaveone-out sensitivity analyses by removing one study at a time and recalculating SMD between groups to confirm that our results were not influenced by a single study.

Assessment of publication bias was done by visual assessment of funnel plots for asymmetry. Begg's rank correlation and Egger's linear regression test were used for quantitative assessment of funnel plots for outcomes containing $\geq 10$ studies.

\section{Results}

Results of the Medline, EMBASE, and Scopus searches and study selection process are shown in Figure 1. A total of 18 observational studies met the inclusion criteria [14-30]. The design and characteristics of the included studies are shown in Table I. Most of the studies had medium to high risk of bias, as presented in Table II.

Sepsis criteria used for participant selection depended on the year of the study, with the majority of studies using the ACCP/SCCM 1992 Consensus Conference definition [31]. Four studies recruited patients with the whole spectrum of sepsis, one study collected data from patients who fulfilled sepsis criteria but did not have organ dysfunction at enrolment, four studies enrolled only patients with septic shock, and nine studies were carried out on a mixed group of patients with severe sepsis and septic shock. The greatest number of studies (12 studies) compared septic patients with healthy volunteers. Other comparison groups included intensive care or hospital patients without inflammation (two studies), patients after cardiac surgery (two studies), and intensive care patients with cardiogenic shock (one study). The median patient age in studies varied from 41 to 72 years, with most patients being in their 50s. There was variation in the proportion of male patients from $24 \%$ to $83 \%$, but gender was not always reported. In eight studies the comparison group was age and gender matched to the study group.

Only in six studies were significant comorbidities (cardiovascular disease, diabetes) affecting vascular function accounted for in the design and analysis of data. In another four studies patients with cardiovascular comorbidity (cardiogenic shock or after cardiac or vascular surgery) were used as a comparison group. There was high risk of confounding because in most studies the groups differed significantly regarding the extent of vasopressor use, sedation, and mechanical ventilation. Two studies included patients receiving nitrates and activated protein C, which are known to influence vascular reactivity measurements.

Methods of evaluation of vascular reactivity were venous occlusion plethysmography with pharmacological or ischaemic provocation, laser Doppler flowmetry using iontophoretically applied or ischaemic provocation, flow-mediated brachial artery vasodilatation measured by ultrasound and passive leg movement and peripheral arterial tonometry (RH-PAT) to obtain the reactive hyperaemia index. One study used more than one method to assess vascular reactivity. Timing of measurements was reported in 10 studies, with seven

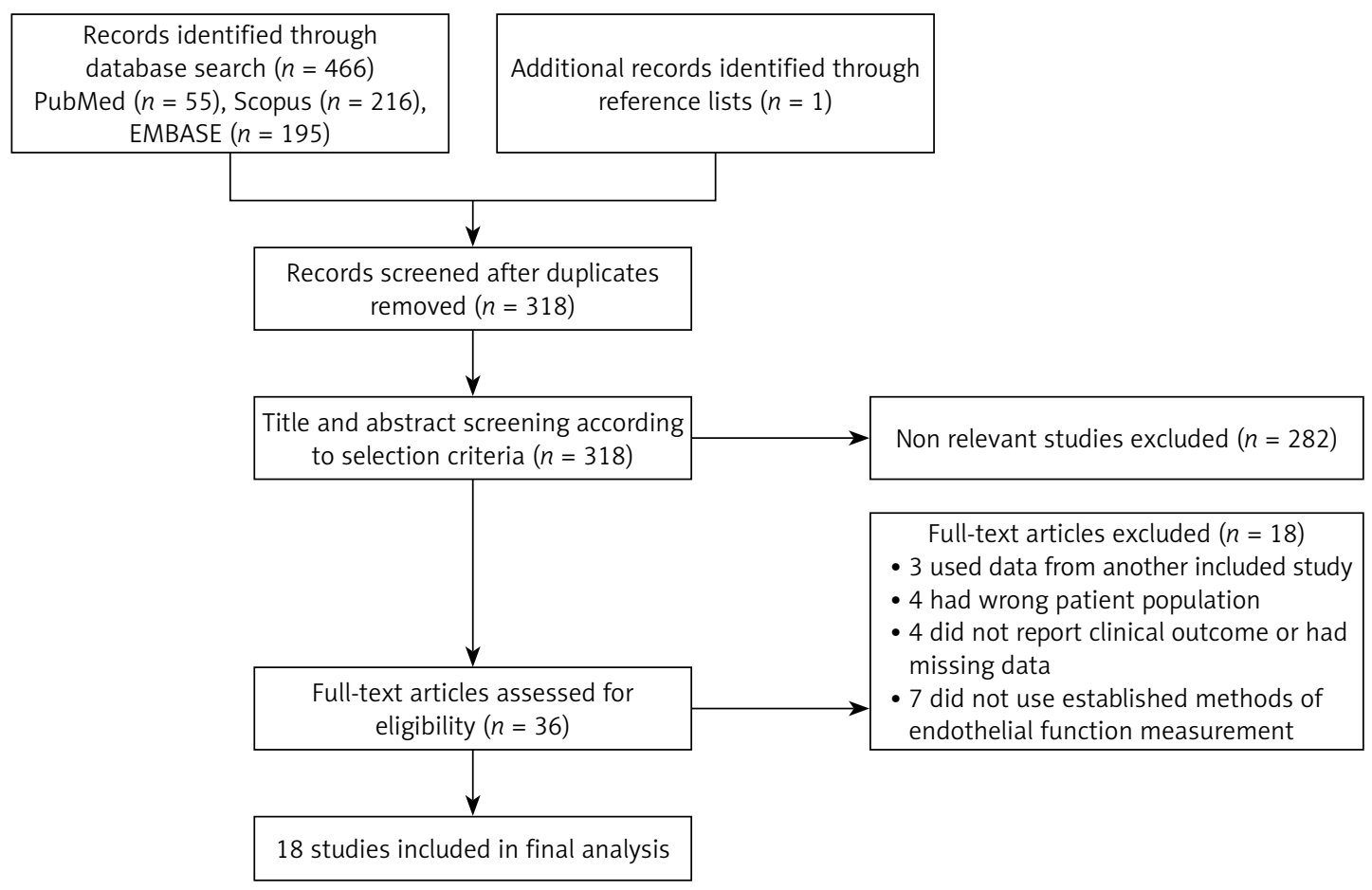

Figure 1. Flow diagram of search process and study selection 


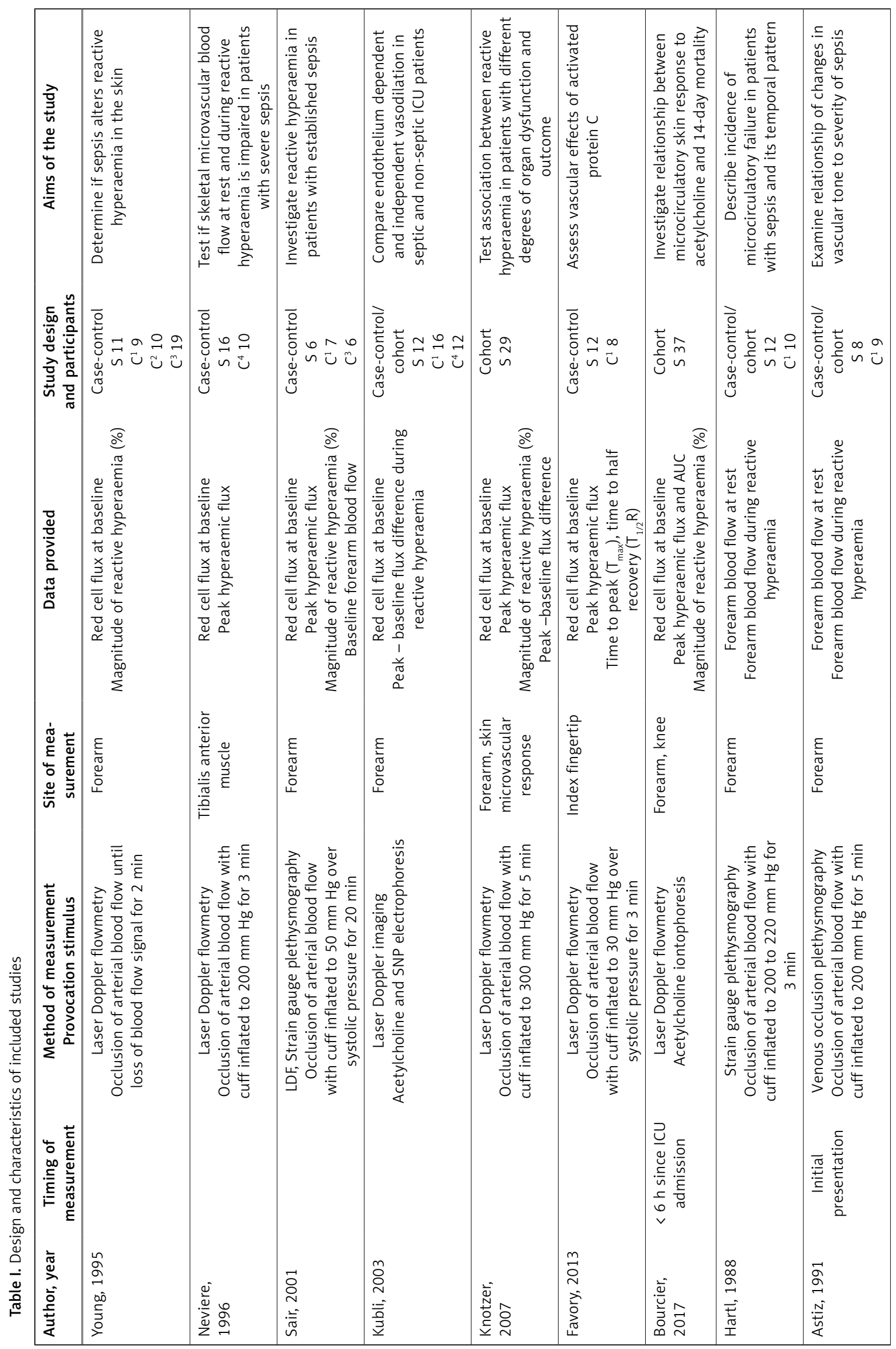




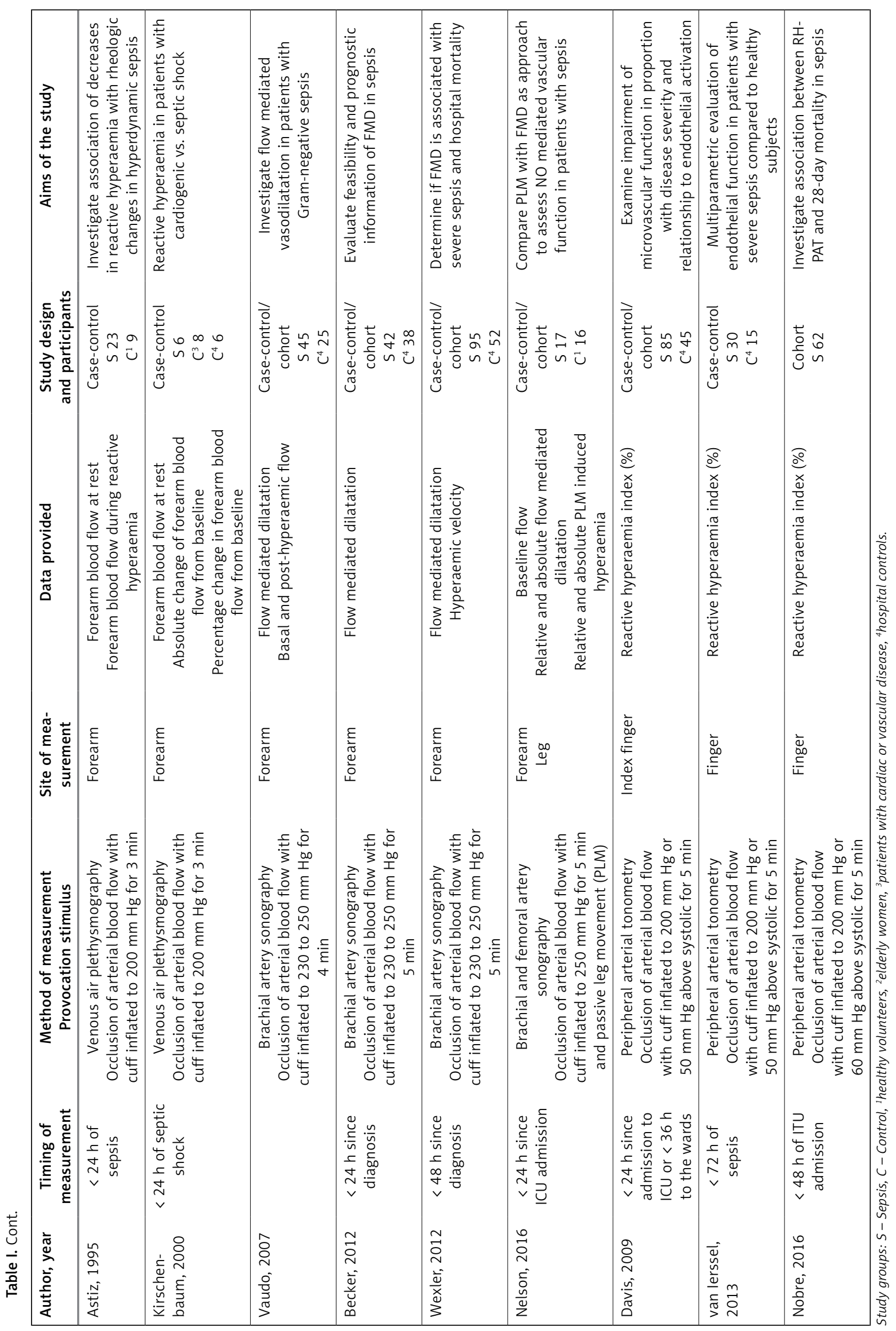


Table II. Summary of risk of bias assessment for the included studies

\begin{tabular}{|c|c|c|c|c|c|c|}
\hline Study & $\begin{array}{l}\text { Representa- } \\
\text { tion }\end{array}$ & Sample size & $\begin{array}{c}\text { Measurement } \\
\text { tool }\end{array}$ & Comparability & $\begin{array}{l}\text { Assessment } \\
\text { of outcome }\end{array}$ & Quality score \\
\hline Young & 0 & 0 & * & * & ** & 4 \\
\hline Neviere & 0 & 0 & * & * & $* *$ & 3 \\
\hline Sair & 0 & 0 & ** & * & $?$ & 3 \\
\hline Knotzer & * & 0 & * & * & * & 5 \\
\hline Kubli & 0 & 0 & * & $* *$ & $* *$ & 5 \\
\hline Favory & 0 & 0 & * & $?$ & $* *$ & 3 \\
\hline Bourcier & * & * & * & 0 & $* *$ & 5 \\
\hline Hartl & 0 & 0 & ** & $?$ & ** & 4 \\
\hline Astiz, 1991 & 0 & 0 & * & * & $* *$ & 4 \\
\hline Astiz, 1995 & 0 & 0 & $* *$ & 0 & $* *$ & 4 \\
\hline Kirschenbaum & 0 & 0 & $* *$ & * & ** & 5 \\
\hline Vaudo & $?$ & * & * & * & $* *$ & 5 \\
\hline Becker & * & * & * & * & $* *$ & 6 \\
\hline Wexler & * & * & * & ** & $* *$ & 7 \\
\hline Nelson & * & 0 & * & $* *$ & ** & 6 \\
\hline Davis & * & * & * & ** & ** & 7 \\
\hline van lerssel & * & 0 & * & * & ** & 5 \\
\hline Nobre & * & * & * & $?$ & $* *$ & 5 \\
\hline
\end{tabular}

One star $\left({ }^{*}\right)$ per category was awarded if sample was representative of the average septic population, sample size was justified and satisfactory, measurement was made with a previously described method, the subjects in different outcome groups were age and gender matched; two stars (**) were awarded for studies that used a gold standard measurement method, controlled for confounding, and assessed outcome reliably; ?, data not available.

studies performing initial measurement within $24 \mathrm{~h}$ of ICU admission or sepsis diagnosis. Eight studies used longitudinal measurements of vascular reactivity performed 24 to $48 \mathrm{~h}$ apart.

The number of patients included in individual studies was small and exceeded 30 in only five studies. Confidence intervals of vasoreactivity measurements were reported in four studies. Overall risk of random error was therefore judged as high. In only two studies, both using peripheral arterial tonometry, was the precision of reactive hyperaemia estimate high.

There were sufficient data in the included studies for statistical pooling for two outcomes, SMD in vasoreactivity between septic patients and controls, and between survivors and non survivors. Measurements of absolute (peak flow) and relative (reactive hyperaemia) change of blood flow or artery diameter after provocation were pooled separately.

Data from 14 studies were included in the analysis of mean difference in vasoreactivity measurements between septic patients and controls. The pooled mean difference estimate from 10 studies including 554 participants showed that septic patients had less reactive hyperaemia than controls $(\mathrm{SMD}=-2.59,95 \% \mathrm{Cl}:-3.46$ to $-1.72 ; Z=5.85$, $p<0.00001$; Figure 2). In nine studies with 354 participants peak hyperaemic blood flow was lower in patients with sepsis than in the control group $(\mathrm{SMD}=-1.42,95 \% \mathrm{Cl}:-2.14$ to $-0.70 ; Z=3.88$, $p=0.0001$; Figure 3). The results of these studies were highly heterogeneous with $R^{2}$ values of $92 \%$ $(p<0.00001)$ and $85 \%(p<0.00001)$, most likely due to differences in study protocols and populations. After removal of any of the studies in the sensitivity analysis the direction of the difference between septic and control group did not change.

Five studies were selected to compare vasoreactivity in survivors and non survivors of sepsis. The combined SMD between non survivors and survivors was -0.36 (95\% Cl: -0.67 to $-0.06 ; Z=$ 2.36; $p=0.02$, Figure 4$)$ for reactive hyperaemia and -0.70 (95\% Cl: -1.13 to $-0.27 ; Z=3.23 ; p=$ 0.001 , Figure 5) for peak hyperaemic blood flow. Both reactive hyperaemia and peak hyperaemic flow were lower in non survivors. Tests for heterogeneity were statistically not significant for both 
estimates. By removing the study by Wexler [23], which had the largest number of participants, statistical significance of difference in peak hyperaemic flow (SMD $=-0.42,95 \% \mathrm{Cl}$ : -0.93 to 0.13 ; $Z=1.5, p=0.13)$ and reactive hyperaemia (SMD $=-0.36,95 \% \mathrm{Cl}:-0.73$ to $0.01 ; Z=1.92 ; p=0.05)$ between survivors and non survivors was lost.

We pooled studies using a control group of patients with severe cardiovascular disease and therefore at high baseline risk of endothelial dysfunction separately. In three studies involving 56 patients the magnitude of reactive hyperaemia in the septic and cardiovascular risk groups was similar (SMD $=-2.23,95 \% \mathrm{Cl}:-4.67$ to 0.21 ; $Z=1.79 ; p=0.07 ; l^{2}=91 \%$, Figure 6).

One study [16] was able to measure endothelium-dependent (EDVD) and -independent vasodilatation separately. No difference in EDVD was found between the septic and control groups.

Conflicting results were reported regarding the relationship between vascular reactivity measurements and development of multiple organ failure (MOF), but the data presented were insufficient for pooling.

Four studies assessed the consistency of findings across different methods of measurement or coherence with biochemical markers. Changes in both macrovascular reactivity measured by venous occlusion plethysmography and microvascular re- activity measured by laser Doppler flowmetry were found in a group of severe sepsis patients by Sair et al. [15]. Three studies assessed coherence of decreased vascular reactivity with biochemical markers of endothelial activation or damage. No correlation was found with levels of endothelin-1 (ET-1) [24], vascular cell adhesion molecule (sVCAM-1) [24], intercellular adhesion molecule (ICAM-1) [26], E selectin [26], endothelial progenitor cells (EPC) [27], and endothelial microparticles [27].

Visual inspection and statistical tests showed asymmetry of the funnel plot examining SMD of reactive hyperaemia in septic patients and controls (Begg's rank correlation test: $p<0.05$; Egger's linear regression test: $p<0.0001$ ), suggesting possible publication bias. No evidence for publication bias was found for other outcomes (Figure 7).

\section{Discussion}

We summarised studies assessing vascular reactivity in patients with sepsis and explored the consistency of association between impaired vascular reactivity and sepsis as well as the ability of functional vascular reactivity tests to predict clinically relevant outcomes.

In the 18 studies using established methods of vascular reactivity measurement included in this review there was convincingly lower vascular reactivity in patients with sepsis compared to controls,

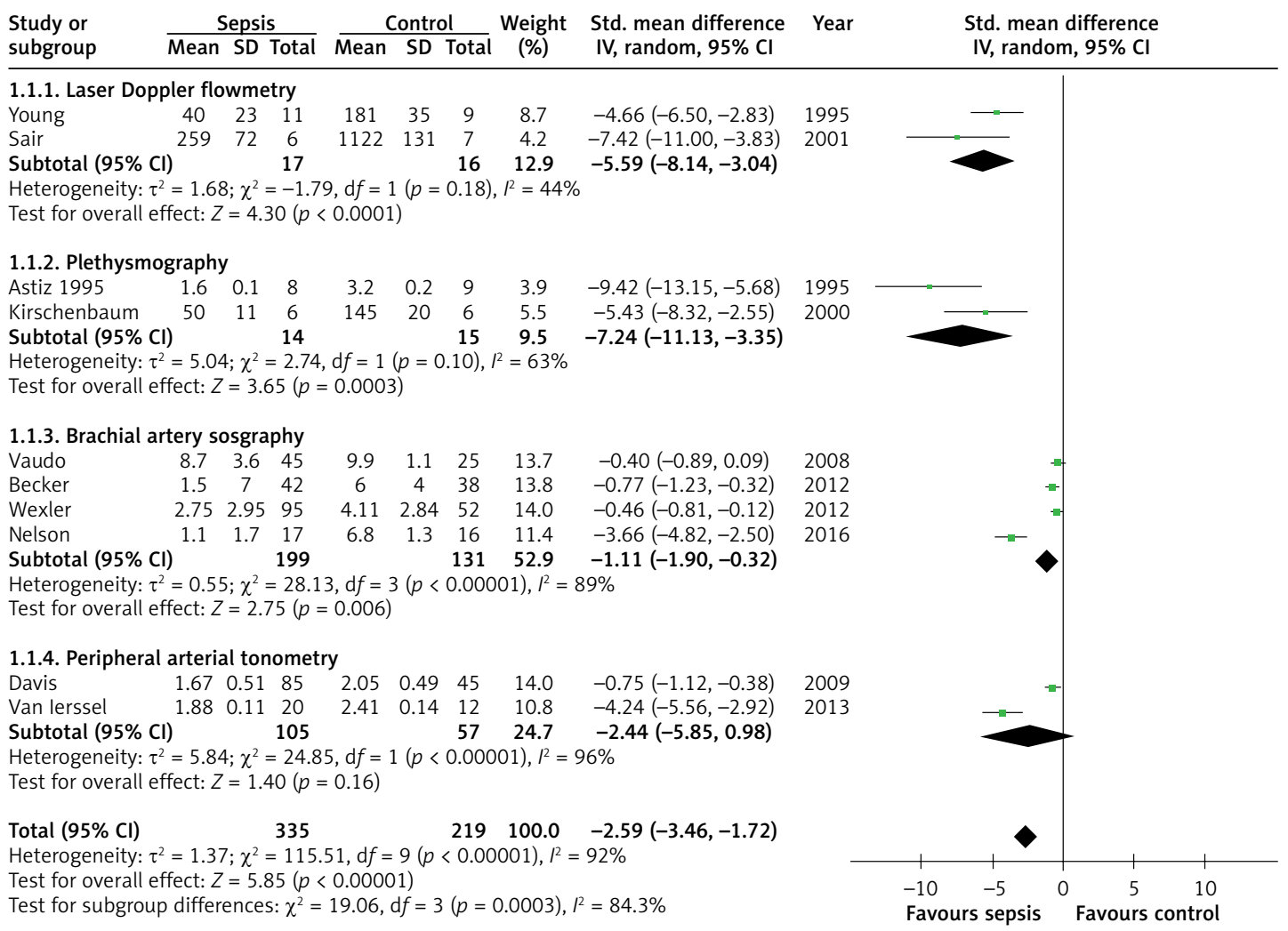

Figure 2. Forest plots of standardised mean difference of reactive hyperaemia in septic patients and controls and subgroup meta-analysis of different methods of measurement 


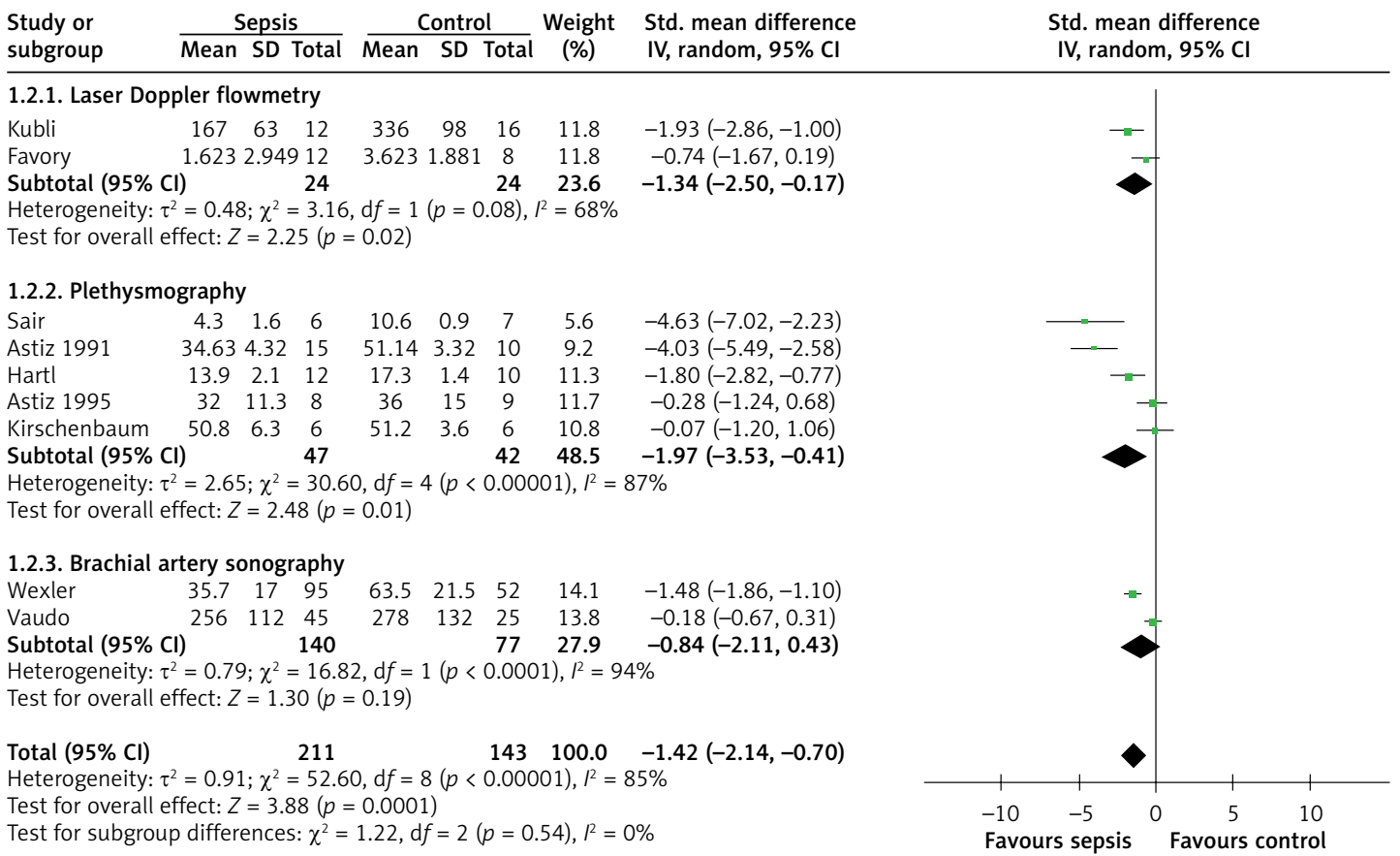

Test for overall effect: $Z=3.88(p=0.0001)$
Test for subgroup differences: $\chi^{2}=1.22, \mathrm{~d} f=2(p=0.54), R^{2}=0 \%$

Favours sepsis Favours control

Figure 3. Forest plots of standardised mean difference of peak hyperaemic flow in septic patients and controls and subgroup meta-analysis of different methods of measurement

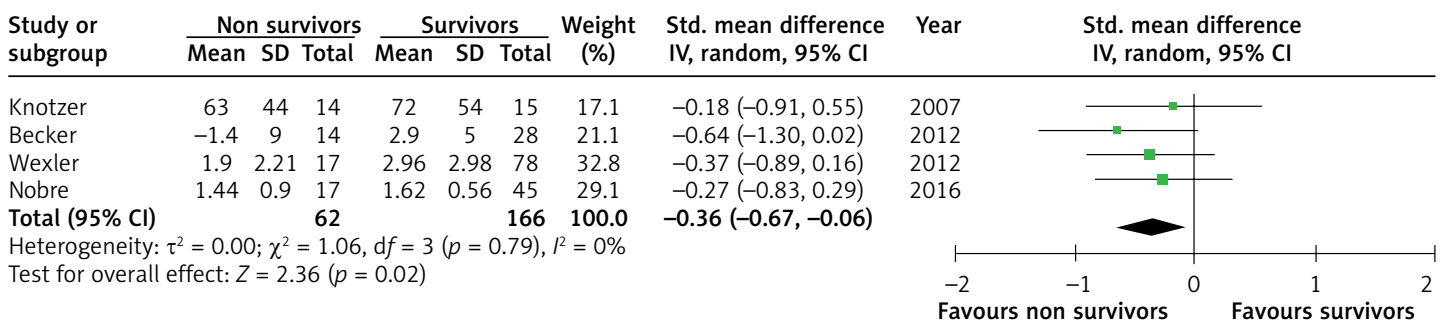

Figure 4. Forest plots of standardised mean difference of reactive hyperaemia in survivors and non survivors of sepsis

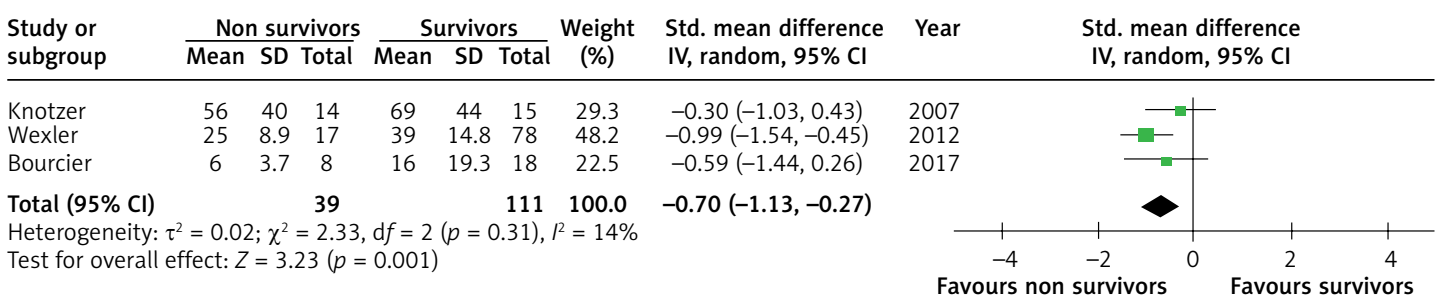

Figure 5. Forest plots of standardised mean difference of peak hyperaemic flow in survivors and non survivors of sepsis

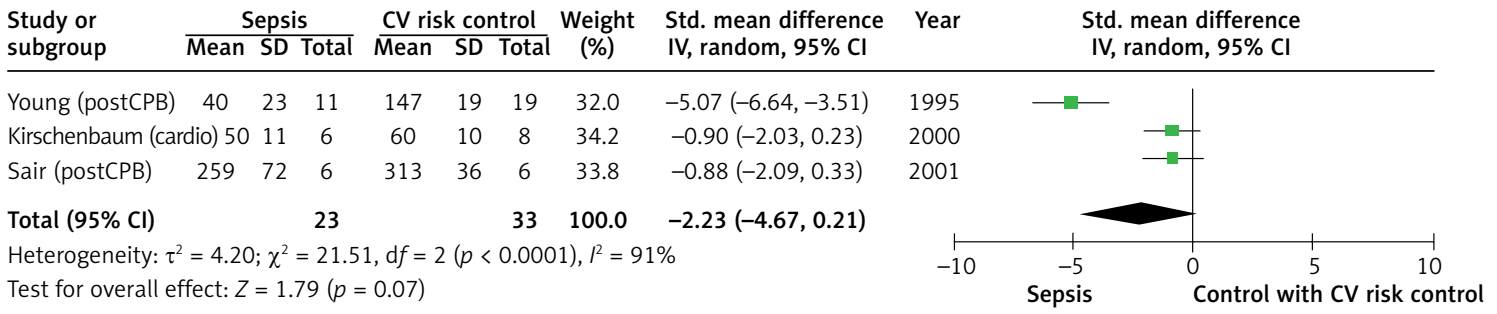

Figure 6. Forest plots of standardised mean difference of reactive hyperaemia in septic patients and controls with

cardiovascular disease 

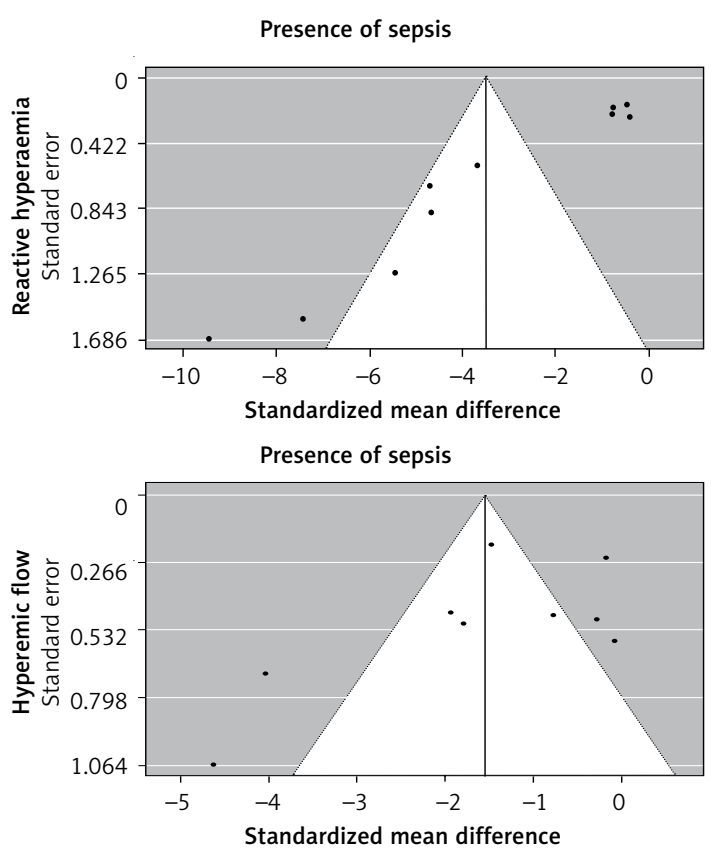

Figure 7. Funnel plots for assessment of publication bias

but the magnitude of the effect was inconsistent across studies. Measures of vascular reactivity in early sepsis were lower in non survivors, but the data were less reliable. There was insufficient data to quantitatively evaluate the relationship between vascular reactivity and development of MOF.

This systematic review is based on a limited number of single site studies using four different measurement methods, many with small sample sizes. There are major differences in the vascular beds explored by the studies - laser Doppler flowmetry evaluates microvascular blood flow, venous air and mercury strain gauge plethysmography allows measurement of total forearm blood flow, flow-mediated vasodilatation (FMD) reflects the bioavailability of endothelium-derived NO in the brachial artery, and RH-PAT measures fingertip reactive hyperaemia. An ideal method of testing vascular reactivity should allow quantification of both endothelium-dependent and -independent vasodilatation. Such methods exist but are invasive and require intraarterial or iontophoretic provocation agent administration, which makes them difficult to use in critically ill patients. The only study using the gold standard research tool [32] - vascular occlusion plethysmography with pharmacological provocation - found no difference in endothelium-dependent vasodilation in patients with septic shock compared with volunteers. Unfortunately, this study had insufficient numerical data to be included in this meta-analysis. Similar results were obtained in studies [16] using measurement of hyperaemia provoked by acetylcholine iontophoresis with laser Doppler flowmetry. Most functional methods used mea-
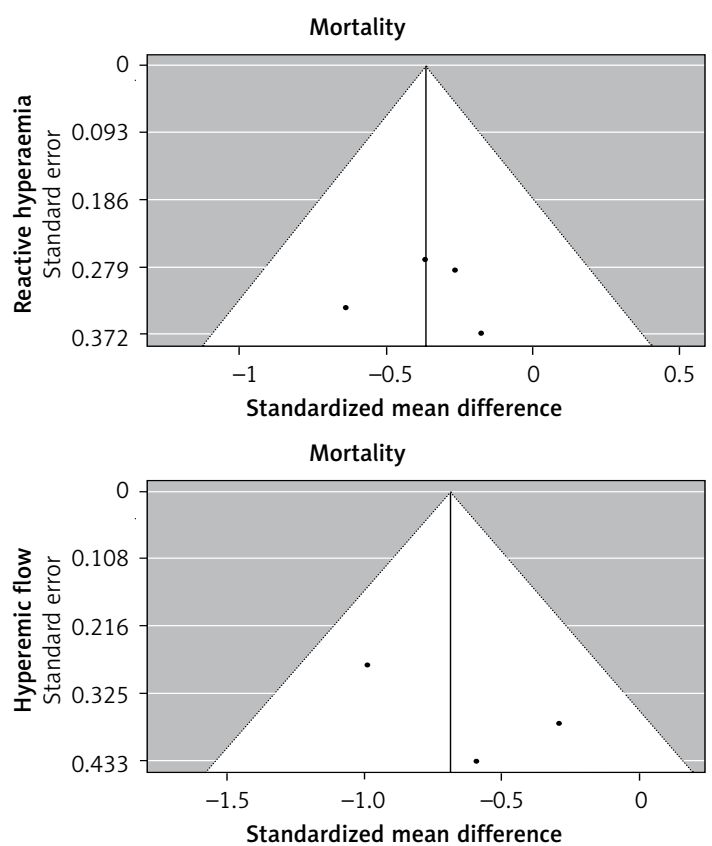

sure a composite value of different mechanisms producing vasodilatation only one of which is bioavailability of $\mathrm{NO}$ and is related to endothelial function; therefore, it is likely that the consistent decrease of vascular reactivity found in sepsis is due to mechanisms other than endothelial dysfunction. Methodological heterogeneity shown by the variety of measurement protocols and sites used in the studies makes findings difficult to generalise.

When comparing the populations from published studies of sepsis epidemiology [33], studies in this review tended to include patients from the same age group but with considerably greater disease severity. Septic patients with a variety of clinical characteristics and in a variety of settings exhibited a decrease in vascular reactivity across studies with the exception of studies that used a comparison group consisting of patients with severe cardiovascular disease. Patients after cardiac and vascular surgery used as comparators have factors other than sepsis, which can influence vascular reactivity and are known to have a particularly high incidence of decreased vascular reactivity [34]. On the other hand, patients with sepsis are often elderly, have advanced atherosclerosis, use statins, have hyperlipidaemia, hypertension, and diabetes, are smokers or obese, and constitute a high-risk group for vascular dysfunction. Only one study in this review enrolled exclusively young patients and another two corrected for comorbidity using the Charlson index. The extent to which comorbidities contribute to results of vascular reactivity tests in septic patients is yet to be established. 
A minority of studies examined changes in vascular reactivity in the context of progression of MOF or mortality. Association with these critical outcomes was not convincing. The reason for the weak of association could be that the pathophysiological mechanisms tested in vascular beds of the forearm do not directly reflect changes in the vasculature involved in splanchnic perfusion. The timing of measurement of endothelial function in the course of sepsis is possibly very relevant. The studies in this review evaluate endothelial function early, within the first 24-48 h of admission, and seek to correlate the extent of changes in vascular reactivity to the progression of sepsis. There might be temporal variation in onset of vascular changes in septic patients, as shown by Hartl et al. [18], who found loss of post-ischaemic hyperaemia to occur on day 8 to 10 from admission in parallel with worsening clinical course and poor prognosis. Longitudinal studies documenting endothelial function from admission to discharge or death would be important to clarify the time course of vascular reactivity and its relation to MOF and mortality.

Experimental and animal data show that sepsis causes endothelial dysfunction, but finding a surrogate marker of endothelial health for use in patients with sepsis is challenging. Other than measurable physiological responses, circulating biomarkers have been investigated in studies. A previous systematic review addressing clinical utility of biomarkers of endothelial activation in sepsis similarly to our review found a correlation between various endothelium-derived molecules and the presence of sepsis, but correlation with clinically important outcomes was not consistent [7]. The reason for inconsistency across studies using different markers has been postulated to be lack of method standardisation, and unclear threshold values and receiver operator characteristics.

This systematic review has several potential limitations. Although we tried to identify all eligible studies by searching three citation databases, only publications in English could be included. There might be studies that have been missed. An important limitation is the small number of included studies; therefore, reliable conclusions could not be drawn about the association between vasoreactivity and development of MOF. Most of the studies were of low methodological quality with a high possibility of random error. Subjects included in both control and sepsis groups were diverse in terms of age, sex, and disease severity, with multiple potential confounders. As none of the studies used random sampling, selection bias is also likely. However, gaps in current knowledge identified by our study could be useful for further research in this area.
Before better conclusions can be drawn the most important first step would be consensus regarding the most suitable method and measurement protocol of vascular reactivity and especially endothelium-dependent vasodilatation for critically ill patients.

In conclusion, from the studies included in this review there is evidence of moderate strength that vascular reactivity is impaired in septic patients, but there is insufficient evidence to suggest that that it is a consequence of endothelial dysfunction or is convincingly related to clinical outcomes.

\section{Conflict of interest}

The authors declare no conflict of interest.

\section{References}

1. Ince C, Mayeux PR, Nguyen T, et al. The endothelium in sepsis. Shock 2016; 45: 259-70.

2. Hack CE, Zeerleder S. The endothelium in sepsis: source of and a target for inflammation. Crit Care Med 2001; 29 (7 Suppl): S21-7.

3. Henneke P, Golenbock DT. Innate immune recognition of lipopolysaccharide by endothelial cells. Crit Care Med 2002; 30 (5 Suppl): S207-13.

4. Aird WC. The role of the endothelium in severe sepsis and multiple organ dysfunction syndrome. Blood 2003; 101: 3765-77.

5. Hawiger J, Veach RA, Zienkiewicz J. New paradigms in sepsis: from prevention to protection of failing microcirculation. J Thromb Haemost 2015; 13: 1743-56.

6. Opal SM, van der Poll T. Endothelial barrier dysfunction in septic shock. J Intern Med 2015; 277: 277-93.

7. Xing K, Murthy S, Liles WC, Singh JM. Clinical utility of biomarkers of endothelial activation in sepsis: a systematic review. Crit Care Lond Engl 2012; 16: R7.

8. Lekakis J, Abraham P, Balbarini A, et al. Methods for evaluating endothelial function: a position statement from the European Society of Cardiology Working Group on Peripheral Circulation. Eur J Cardiovasc Prev Rehabil 2011; 18: 775-89.

9. Liberati A, Altman DG, Tetzlaff J, et al. The PRISMA statement for reporting systematic reviews and meta-analyses of studies that evaluate healthcare interventions: explanation and elaboration. BMJ 2009; 339: b2700.

10. Wells G, Shea B, O'Connell D, Peterson D, Welch V, Losos M. The Newcastle-Ottawa Scale (NOS) for assessing the quality of nonrandomised studies in meta-analyses [Internet]. 2018. Available from: http://www.ohri.ca/ programs/clinical_epidemiology/oxford.asp.

11. Higgins JPT, Green S (editors). Cochrane Handbook for Systematic Reviews of Interventions Version 5.1.0 [updated March 2011]. The Cochrane Collaboration, 2011. Available from http://handbook.cochrane.org.

12. DerSimonian R, Laird N. Meta-analysis in clinical trials. Control Clin Trials 1986; 7: 177-88.

13. Higgins JPT, Thompson SG, Deeks JJ, Altman DG. Measuring inconsistency in meta-analyses. BMJ 2003; 327: 557-60.

14. Young JD, Cameron EM. Dynamics of skin blood flow in human sepsis. Intensive Care Med 1995; 21: 669-74.

15. Sair M, Etherington PJ, Peter Winlove C, Evans TW. Tissue oxygenation and perfusion in patients with systemic sepsis. Crit Care Med 2001; 29: 1343-9. 
16. Kubli S, Boëgli Y, Ave AD, et al. Endothelium-dependent vasodilation in the skin microcirculation of patients with septic shock. Shock 2003; 19: 274-80.

17. Favory R, Poissy J, Alves I, et al. Activated protein C improves macrovascular and microvascular reactivity in human severe sepsis and septic shock. Shock 2013; 40: 512-8.

18. Hartl WH, Günther B, Inthorn D, et al. Reactive hyperemia in patients with septic conditions. Surgery 1988; 103: 440-4.

19. Astiz ME, Tilly E, Rackow ED, Weil MH. Peripheral vascular tone in sepsis. Chest 1991; 99: 1072-5.

20. Astiz ME, DeGent GE, Lin RY, Rackow EC. Microvascular function and rheologic changes in hyperdynamic sepsis. Crit Care Med 1995; 23: 265-71.

21. Kirschenbaum LA, Astiz ME, Rackow EC, Saha DC, Lin R. Microvascular response in patients with cardiogenic shock. Crit Care Med 2000; 28: 1290-4.

22. Vaudo G, Marchesi S, Siepi D, et al. Human endothelial impairment in sepsis. Atherosclerosis 2008; 197: 747-52.

23. Wexler O, Morgan MAM, Gough MS, et al. Brachial artery reactivity in patients with severe sepsis: an observational study. Crit Care Lond Engl 2012; 16: R38-8.

24. Becker L, Prado K, Foppa M, et al. Endothelial dysfunction assessed by brachial artery ultrasound in severe sepsis and septic shock. J Crit Care 2012; 27: 316.e9-14.

25. Nelson AD, Rossman MJ, Witman MA, et al. Nitric oxide-mediated vascular function in sepsis using passive leg movement as a novel assessment: a cross-sectional study. J Appl Physiol (1985) 2016; 120: 991-9.

26. Davis JS, Yeo TW, Thomas JH, et al. Sepsis-associated microvascular dysfunction measured by peripheral arterial tonometry: an observational study. Crit Care 2009; 13: R155-5.

27. van lerssel SH, Van Craenenbroeck EM, Hoymans VY, Vrints CJ, Conraads VM, Jorens PG. Endothelium dependent vasomotion and in vitro markers of endothelial repair in patients with severe sepsis: an observational study. PLoS One 2013; 8: e69499-9.

28. Knotzer H, Maier S, Dünser M, et al. Oscillation frequency of skin microvascular blood flow is associated with mortality in critically ill patients. Acta Anaesthesiol Scand 2007; 51: 701-7.

29. Bourcier S, Joffre J, Dubée V, et al. Marked regional endothelial dysfunction in mottled skin area in patients with severe infections. Crit Care 2017; 21: 155.

30. Nobre V, Ataíde TB, Brant LC, et al. Use of reactive hyperemia - peripheral arterial tonometry and circulating biological markers to predict outcomes in sepsis. Rev Bras Ter Intensiva 2016; 28: 387-96.

31. Bone RC, Balk RA, Cerra FB, et al. Definitions for sepsis and organ failure and guidelines for the use of innovative therapies in sepsis. The ACCP/SCCM Consensus Conference Committee. American College of Chest Physicians/Society of Critical Care Medicine. Chest 1992; 101: 1644-55.

32. Kienbaum P, Prante C, Lehmann N, Sander A, Jalowy A, Peters J. Alterations in forearm vascular reactivity in patients with septic shock. Anaesthesia 2008; 63: 121-8.

33. Stoller J, Halpin L, Weis M, et al. Epidemiology of severe sepsis: 2008-2012. J Crit Care 2016; 31: 58-62.

34. Bellamkonda K, Williams M, Handa A, Lee R. Flow mediated dilatation as a biomarker in vascular surgery research. J Atheroscler Thromb 2017; 24: 779-87. 